\title{
Ergebnis einer integrativen Behandlung bei stationären Patienten mit chronischen Leiden - eine Beobachtungsstudie
}

\author{
Cesar Winnicki Rahel Ernst \\ Aeskulap Klinik, Brunnen, Schweiz
}

\section{Schlüsselwörter \\ Komplementärmedizin · Integrative Behandlung · Wirksamkeit . Ergebnismessung $\cdot$ Lebensqualität $\cdot$ SF-12}

\section{Zusammenfassung}

Hintergrund: Beurteilung der subjektiven Ergebnisse (Outcome) einer stationären, integrativen Behandlung bei Patienten mit diversen chronischen Erkrankungen im Sinne gesundheitsbezogener Lebensqualität (LQ). Methoden: Eine systematische Erhebung der gesundheitsbezogenen LQ wurde in der Aeskulap Klinik, Zentrum für ärztliche Ganzheitsmedizin, Brunnen, Schweiz, bei allen stationären Patienten bei Eintritt, Austritt sowie 4 Wochen nach der Hospitalisation mittels des Fragebogens SF-12 durchgeführt. Die erfassten Daten erlaubten eine statistische Beurteilung sowohl der initialen gesundheitlichen Situation als auch des Behandlungsergebnisses hinsichtlich körperlicher (KSS) und psychischer (PSS) Gesundheit. Ergebnisse: Es wurden insgesamt 194 stationäre Patienten beurteilt, wobei die Rücklaufquote für die Erhebung während der Hospitalisation $83,0 \%$ und für die poststationäre Erhebung $54,1 \%$ betrug. Für das gesamte Kollektiv wurde eine wesentliche, statistisch signifikante Verbesserung sowohl des körperlichen als auch des psychischen Gesundheitszustands festgestellt. Der initiale KSS-Wert von $31,49 \pm 11,2$ verbesserte sich um 5,83 $\pm 10,1$ Skalenpunkte während der Hospitalisation sowie um 7,03 \pm 9,9 Skalenpunkte danach, was einer Effektgrösse von 0,54 bzw. 0,68 entsprach ( $p<0,0001$ bzw. $p<$ $0,0001)$. Der psychische PSS-Wert stieg vom Ausgangswert 33,87 \pm 13,3 um 9,3 $\pm 12,6$ bzw. 9,9 $\pm 14,4$ an (Effektgrösse 0,74 bzw. 0,87; $p<$ $0,0001$ bzw. $p<0,0001)$. Über $60 \%$ aller Patienten erreichten einen Anstieg der LQ-Parameter von über 5 Skalenpunkten (klinisch relevantes Ergebnis). Schlussfolgerungen: Vor dem Hintergrund der chronischen, komplexen Erkrankungen der behandelten Patienten deuten die erzielten Ergebnisse der integrativen stationären Behandlung auf einen klinisch relevanten Behandlungserfolg hin. Das Problem der geringen Kollektivgrösse, des Datenverlusts bei der poststationären Befragung sowie des kurzen Follow-ups begrenzen die Aussagekraft dieser Beobachtungsstudie.

(c) 2015 S. Karger GmbH, Freiburg

\begin{abstract}
Keywords
CAM - Complementary medicine - Integrative treatment - Effectiveness . Outcome measurement $\cdot$ Quality of life $\cdot$ SF-12
\end{abstract}

\section{Summary}

Results of an Integrative Treatment Approach for In-Patients with Chronic Diseases - an Observational Study

Background: This observational study explores the influence of an integrative treatment approach during the hospitalisation of patients with different chronic diseases in terms of their health-related quality of life (QoL). Methods: Systematic measurements based on the health-related QoL questionnaire SF-12 were carried out in the Aeskulap Klinik, Centre for Holistic Medicine, Brunnen, Switzerland, for all in-patients on admission, at discharge, and 4 weeks after their discharge from hospital. The acquired data enabled a statistical assessment of the initial health state of the patients as well as of the treatment outcome concerning physical (KSS) and mental (PSS) health. Results: 194 in-patients were involved in the study. The feedback obtained during the in-patient stay was $83.0 \%$ and amounted to $54.1 \%$ for all three measurements (including 4 weeks after discharge). A considerable and significant statistical improvement of both physical and mental health was observed in the whole group of patients. The initial KSS value of $31.49 \pm 11.2$ improved by $5.83 \pm 10.1$ points during hospitalization and by $7.03 \pm 9.94$ weeks later, which correlates with an effect size of $0.54(p<0.0001)$ and $0.68(p<$ 0.0001 ), respectively. The mental PSS increased from $33.87 \pm 13.3$ by $9.3 \pm 12.6$ and by $9.9 \pm 14.4$ (effect size $0.74, p<0.0001$ and 0.87 , $p<$ 0.0001 , respectively). An increase of QoL parameters $>5$ points (clinically relevant outcome) was stated by $>60 \%$ of our patients. Conclusion: Taking into account the chronic und complex diseases of our patients, the obtained results suggest a clinically relevant success under integrative in-patient treatment. Limitations regarding the conclusiveness of our study are the problem of a low number of patients, data loss in follow-up queries, and the short follow-up period.

(c) 2015 S. Karger GmbH, Freiburg

\section{KARGER \\ Fax +497614520714} Information@Karger.com www.karger.com
Dr. med. Cesar Winnicki

Rubisacherrain 15,6440 Brunnen, Schweiz cesar.winnicki@bluewin.ch 


\section{Einleitung}

Komplementärmedizin erfreut sich nicht nur in der Schweiz einer grossen Beliebtheit und Akzeptanz. Die aktuelle Gesetzgebung (Krankenversicherungsgesetz) verlangt, dass alle erbrachten Versorgungsleistungen wirksam, zweckmässig und wirtschaftlich sein müssen. Der Nachweis der Wirksamkeit vieler komplementärmedizinischer Massnahmen wurde bis anhin noch nicht ausreichend geführt.

Das Patientengut der Komplementärmedizin fällt durch Inhomogenität und Polymorbidität auf; vorzugsweise werden chronische Erkrankungen behandelt. Darüber hinaus beinhaltet die komplementärmedizinische Betreuung ein breites Spektrum an Methoden, die individuell und meistens in Kombination eingesetzt werden. Diese Vielfalt und Komplexität der ganzheitlichen Betreuung erklärt, warum der Wirksamkeitsnachweis schwierig ist $[1,2]$. Vor diesem Hintergrund gewann in den letzten Jahren die gesundheitsbezogene Lebensqualität (LQ) als klinisches Instrument für Ergebnismessung an Bedeutung $[3,4]$. Diese generischen Instrumente sind dahingehend konzipiert, dass sie unabhängig von einer bestimmten Erkrankung die LQ der Betroffenen quantifizieren und somit eine Vergleichbarkeit der Ergebnisse über verschiedene Patientengruppen und medizinische Behandlungsmassnahmen hinweg erlauben $[5,6]$.

Die private, gemeinnützige Aeskulap Klinik (AK), Zentrum für Ärztliche Ganzheitsmedizin, in Brunnen, Schweiz, behandelt seit ihrer Gründung im Jahr $1990 \mathrm{~Pa}$ tienten mit diversen chronischen Erkrankungen im Sinne eines integrativen Therapiekonzepts. AK ist durch die kantonalen Gesundheitsbehörden offiziell als eine Spezialklinik anerkannt. Methoden der konventionellen Medizin werden individuell mit diversen Methoden der ärztlichen Komplementärmedizin kombiniert - mit dem Ziel, ein möglichst optimales und nachhaltiges Ergebnis zu erreichen. Das medizinische Angebot der AK fokussiert sich vorwiegend auf folgende Bereiche: chronische Erkrankungen der Atemwege, des Verdauungstrakts sowie kardiovaskuläre Erkrankungen, Onkologie, Psychosomatik und Schmerztherapie. Zu den wichtigsten komplementärmedizinischen Behandlungsmethoden zählen: Traditionelle Chinesische Medizin inklusive Akupunktur, Neuraltherapie, Homöopathie, Phytotherapie, orthomolekulare Medizin, Probiotika, Sauerstofftherapien und Hyperthermie. Ansätze aus dem Bereich der Mind-BodyMedizin wie Psychotherapie, Physiotherapie und Ernährungskonzepte ergänzen das Therapieangebot. Um die Ergebnisse der integrativen Betreuung quantitativ zu beurteilen, wird bei allen stationären Patienten eine systematische Erhebung der gesundheitsbezogenen LQ mittels des Fragebogens SF-12 (Short Form 12) zu Beginn und nach der Hospitalisation durchgeführt.
Das Ziel der vorliegenden Arbeit besteht darin, die subjektiven Ergebnisse (Outcome) einer integrativen Behandlung bei stationären Patienten mit diversen chronischen Erkrankungen im Sinne der gesundheitsbezogenen LQ zu beurteilen. Da die angewandten Therapiekonzepte sehr individuell und vor allem vom Krankheitsbild abhängig waren, ging es darum, eine globale Aussage über die subjektive Wahrnehmung der Patienten in Bezug auf ihre LQ vor und nach der Hospitalisation (whole system evaluation) zu erhalten. Die Untersuchung fand im Rahmen eines multimodalen Qualitätsmanagements der AK statt.

\section{Patienten und Methoden}

\section{Studiendesign und Setting}

Es handelt sich um eine kontinuierliche prospektive Beobachtungsstudie zur Beurteilung des subjektiven Behandlungsergebnisses während und 4 Wochen nach einer stationären Behandlung in der AK, einer Privatklinik für Ärztliche Ganzheitsmedizin, in Brunnen, Schweiz. Die AK verfügt neben dem grossen Ambulatorium über 26 stationäre Betten und ist vollständig in das Schweizerische Gesundheitswesen integriert.

Bei allen stationären Patienten der AK kam ein integratives Therapiekonzept zum Einsatz, das aus folgenden Elementen bestand:

- konventionelle Massnahmen im Sinne der evidenzbasierten Medizin (Schulmedizin);

- komplementärmedizinische Massnahmen;

- Elemente aus der Mind-Body-Medizin in Form von Psychotherapie, Be wegungs- und Physiotherapie sowie Ernährung.

Die Erhebung der gesundheitsbezogenen LQ erfolgte anhand des Fragebogens SF-12 jeweils bei der Aufnahme, bei der Entlassung und 4 Wochen poststationär.

\section{Patienten}

Die vorliegenden Daten beziehen sich auf die Zeitperiode vom 1. Oktober 2011 bis zum 31. März 2013, in der 194 chronisch kranke, vorwiegend poly morbide Patienten behandelt und evaluiert wurden. $\mathrm{Zu}$ den wichtigsten Krankheitsbildern gehörten psychosomatische, rheumatologische und onkologische Leiden. Von der Auswertung wurden Patienten mit einer Hospitalisationsdauer von weniger als 5 Tagen ausgeschlossen. Alle Patienten wurden schriftlich über den Zweck der Erhebung informiert und gaben ihre Zustimmung für die anonymisierte Auswertung ihrer Daten.

\section{Interventionen}

Aus dem breiten Fundus an komplementärmedizinischen Methoden, die den Patienten der AK zur Verfügung stehen, wurde jeweils ein individuelles Therapiekonzept erstellt und durchgeführt. Die Basis für dieses Konzept bildeten eine ausführliche Anamnese sowie eine konventionelle und komplementäre Diagnostik. Die komplementäre Diagnostik beinhaltete in der Regel folgende Tests: Thermoregulationsdiagnostik nach Rost, Herzratenvariabilität, Panoramaschichtaufnahme des Gebisses (OPT) sowie in ausgewählten Fällen Bioelektronik nach Vincent und eine Darmflorauntersuchung. Bei pathologischem Befund der Panoramaschichtaufnahme des Zahn-Kiefer-Bereichs vor allem im Sinne einer dentogenen Beherdung erfolgte eine ganzheitliche zahnärztliche Beratung und allenfalls eine Behandlung in der klinikinternen Abteilung für Orale Medizin.

Im Sinne der komplementärmedizinischen Behandlung wurden folgende Methoden eingesetzt: Traditionelle Chinesische Medizin inklusive Akupunktur (bei 56,2\% aller Patienten), Neuraltherapie oder therapeutische Lokalanästhesie (37,3\%), Homöopathie (75,2\%), Phytotherapie $(98,5 \%)$, orthomolekulare Medizin (86,7\%), Probiotika (80,5\%), Sauerstofftherapien $(88,4 \%)$ und Hyperthermie $(22,2 \%)$. Das integrative Konzept wurde durch 
Tab. 1. Vergleich der Responder mit Non-Respondern

\begin{tabular}{|c|c|c|c|c|c|}
\hline & \multirow[t]{2}{*}{ ICD-10 } & \multicolumn{2}{|c|}{ Responder } & \multicolumn{2}{|c|}{ Non-Responder } \\
\hline & & $\mathrm{n}$ & $\%$ & $\mathrm{n}$ & $\%$ \\
\hline \multicolumn{6}{|l|}{ Diagnose } \\
\hline Neubildungen & $\mathrm{C} 00-\mathrm{C} 48$ & 20 & 12,4 & 15 & 45,5 \\
\hline Affektive Störungen & F30-F39 & 51 & 31,7 & 6 & 18,2 \\
\hline $\begin{array}{l}\text { Neurotische und somatoforme } \\
\text { Störungen }\end{array}$ & F40-F48 & 23 & 14,3 & & \\
\hline Essstörungen & F50 & 3 & 1,9 & 1 & 3 \\
\hline $\begin{array}{l}\text { Migräne, Kopfschmerzen, } \\
\text { Schmerzen allgemein }\end{array}$ & $\begin{array}{l}\text { G43-G44 } \\
\text { R51-R52 }\end{array}$ & 13 & 8 & 2 & 6 \\
\hline Arthropathien & M00-M25 & 11 & 6,8 & 3 & 9,1 \\
\hline Erkrankungen der Wirbelsäule & M40-M54 & 7 & 4,3 & 3 & 9,1 \\
\hline Kollagenosen & M30-M36 & 4 & 2,5 & & \\
\hline Sonstiges Rheuma & $\mathrm{M}$ & 6 & 3,7 & & \\
\hline Sonstige Erkrankungen & & 23 & 14,3 & 3 & 9,1 \\
\hline Alle & & 161 & 100 & 33 & 100 \\
\hline \multirow[t]{2}{*}{ Verstorben (bis 09/2013) } & & 2 & 1,2 & 9 & 27,2 \\
\hline & & \multicolumn{2}{|c|}{ Responder } & \multicolumn{2}{|c|}{ Non-Responder } \\
\hline \multicolumn{6}{|l|}{ Allgemeines } \\
\hline Alter, Jahre & & \multicolumn{2}{|c|}{$57,9 \pm 14,9$} & \multicolumn{2}{|c|}{$58,1 \pm 15,2$} \\
\hline Weibliches Geschlecht, \% & & \multicolumn{2}{|c|}{82,0} & \multicolumn{2}{|c|}{54,5} \\
\hline Erkrankungsdauer, Jahre & & \multicolumn{2}{|c|}{$9,2 \pm 7,8$} & \multicolumn{2}{|c|}{$6,4 \pm 4,8$} \\
\hline Verweildauer, Tage & & \multicolumn{2}{|c|}{$21,4 \pm 9,8$} & \multicolumn{2}{|c|}{$18,0 \pm 12,6$} \\
\hline
\end{tabular}

Psychotherapie und Entspannungsverfahren (65,4\%) sowie Physiotherapie $(97,1 \%)$ in ihrer ganzen Breite mit einem besonderen Augenmerk auf die Rekonditionierung und Beratung bezüglich körperlicher Aktivität ergänzt. Das verwendete Therapiekonzept war durch eine hohe Therapiedichte gekennzeichnet. Alle Patienten erhielten neben den regelmässigen ärztlichen Konsultationen (mindestens dreimal pro Woche) mindestens zwei verschiedene Massnahmen pro Tag. Beispielsweise erfolgten Physiotherapie sechsmal, Sauerstofftherapien dreimal und Psychotherapie mindestens zweimal pro Woche. Alle Patienten erhielten Vollwertkost ohne Fleisch und je nach Bedarf eine Ernährungsberatung.

\section{Datenerhebung und Outcome-Messung}

Basierend auf der klassischen Definition von Gesundheit der Weltgesundheitsorganisation (WHO), muss sich die gesundheitsbezogene LQ auf körperliche, psychische und soziale Aspekte der menschlichen Existenz beziehen. Entscheidend dabei ist, dass diese Aspekte von Patienten subjektiv beurteilt und geäussert werden. Generell lassen sich die Instrumente zur Messung der gesundheitsbezogenen LQ in krankheitsübergreifende (generische) oder krankheitsspezifische Instrumente unterteilen [4].

Die Beurteilung der gesundheitsbezogenen LQ unserer Patienten erfolgte mit dem generischen Fragebogen SF-12. Der SF-12 ist die validierte Kurzversion des seit den 1980er-Jahren bekannten Fragenbogens SF-36 und beinhaltet lediglich 12 Items. Der SF-12 erlaubt eine verlässliche Ermittlung der körperlichen und psychischen LQ als körperliche und psychische Summenskalen (KSS bzw. PSS). Ein Anstieg des Skalenergebnisses von mehr als 5 Skalenpunkten gilt als klinisch relevant. Normdaten für den deutschsprachigen SF-12 wurden bei der deutschen Bevölkerung erhoben und erarbeitet [7].

Alle Patienten erhielten bei Eintritt, bei Austritt und 4 Wochen nach der Entlassung einen patientenbezogenen Fragebogen SF-12, der von den meisten Patienten selbst ausgefüllt wurde. In Ausnahmefällen, vor allem in Bezug auf die poststationäre Erfassung, erfolgte die Erhebung durch ein telefonisches Interview durch den Assistenzarzt. Der Fragebogen wurde in das Klinikinformationssystem integriert, und die Berechnung der Summenskalen (Umcodierung, Erstellung von Dummy-Variablen, Gewichtung und Stan- dardisierung) erfolgte elektronisch nach der in der Quelle angegebenen Methodik [7]. Die Benutzung des SF-12 wurde vom Rechtseigentümer Hogrefe Verlag GmbH \& Co. aus Göttingen, Deutschland, genehmigt.

Über das klinikinterne Informationssystem wurden Angaben zu Hauptund Nebendiagnosen (ICD-10) sowie alle weiteren Daten (Demographie, Therapieprofil) abgerufen. Bei der Zuteilung der Patienten zu den definierten Diagnosegruppen wurde die bei der Entlassung gestellte Hauptdiagnose verwendet.

\section{Statistische Methoden}

Die statistische Auswertung aller erhobenen Daten erfolgte rein deskriptiv durch Berechnung statistischer Kerngrössen wie arithmetischer Mittelwert (M) und Standardabweichung (SD). Die Unterschiede zwischen verbundenen Stichproben wurden mit dem t-Test (univariable Statistik) und die Normalverteilung der Stichproben mit dem Chi-Quadrat-Test geprüft.

Die Darstellung der Veränderungsmassen (mittlere Differenz zwischen Vor- und Nach-Werten) wurde durch Berechnung von Effektgrössen (d) gemäss der Methodik von Dunlop [8] standardisiert, wobei $\mathrm{d}=0,2$ einen kleinen, $d=0,5$ einen mittleren und $d=0,8$ einen starken Effekt bedeutet. Folgende Formel wurde benutzt:

$$
\mathrm{d}=\frac{\mathrm{M}_{1}-\mathrm{M}_{2}}{\sqrt{\frac{1}{2}\left(S D_{1}^{2}+S D_{2}^{2}\right)}}
$$

Die Datenanalyse erfolge mithilfe des Programms MATLAB (The Math Works, Inc., Natick, MA, USA).

\section{Ergebnisse}

\section{Response}

Zwischen dem 1. Oktober 2011 und dem 31. März 2013 wurden in der AK insgesamt 199 Patientinnen und Pati- 
Tab. 2. Erhebungsdaten für alle Responder $(\mathrm{n}=161)$

\begin{tabular}{|c|c|c|c|c|c|c|c|}
\hline \multirow[t]{2}{*}{$\mathrm{n}$} & \multirow[t]{2}{*}{ Eintritt } & \multirow[t]{2}{*}{ Austritt } & \multirow[t]{2}{*}{4 Wochen } & \multirow[t]{2}{*}{ Differenz } & \multirow[t]{2}{*}{$\mathrm{p}$} & \multicolumn{2}{|c|}{ Effektgrösse } \\
\hline & & & & & & $\mathrm{d}$ & $\geq 5$ Punkte, $\%$ \\
\hline \multicolumn{8}{|c|}{ Körperliche Gesundheit (Körperliche Summenskala, KSS) $M \pm S D$} \\
\hline 161 & $31,49 \pm 11,2$ & $37,33 \pm 10,4$ & - & $5,83 \pm 10,1$ & $<0,0001$ & 0,54 & 55,9 \\
\hline 105 & $30,44 \pm 10,4$ & - & $37,47 \pm 10,3$ & $7,03 \pm 9,9$ & $<0,0001$ & 0,68 & 61,9 \\
\hline 105 & - & $36,22 \pm 10,7$ & $37,47 \pm 10,3$ & $1,26 \pm 7,9$ & 0,107 & 0,12 & \\
\hline \multicolumn{8}{|c|}{ Psychische Gesundheit (Psychische Summenskala, PSS) $M \pm S D$} \\
\hline 161 & $33,87 \pm 13,3$ & $43,14 \pm 11,7$ & - & $9,3 \pm 12,6$ & $<0,0001$ & 0,74 & 64,0 \\
\hline 105 & $35,28 \pm 11,2$ & - & $45,14 \pm 11,4$ & $9,9 \pm 14,4$ & $<0,0001$ & 0,87 & 67,6 \\
\hline 105 & - & $43,97 \pm 11,0$ & $45,14 \pm 11,4$ & $1,2 \pm 11,0$ & 0,275 & 0,1 & \\
\hline
\end{tabular}

enten hospitalisiert. An der Untersuchung nahmen 194 (97,5\%) hiervon teil; die 5 restlichen Patienten wurden kürzer als 5 Tage hospitalisiert (Ausschlusskriterium).

161 (83,0\%) untersuchte Patienten (Responder) gaben Daten zur Outcome-Messung ab, 105 (54,1\%) davon füllten alle drei Erhebungen aus. Bei 33 (17,0\%) Patienten erhielten wir keine Angaben (Non-Responder). In Tabelle 1 werden die Responder und Non-Responder miteinander verglichen. Die Non-Responder $(\mathrm{n}=33)$ unterschieden sich von den Respondern durch eine kürzere Erkrankungsdauer und Hospitalisationszeit, eine unterschiedliche Verteilung der Hauptdiagnosen sowie eine höhere Mortalität (bis September 2013). Bei den NonRespondern hatten 45,5\% onkologische Diagnosen, und $27,2 \%$ dieser Patientengruppe verstarben innerhalb von 6 Monaten nach der Behandlung.

\section{Hauptdiagnosen}

Die Verteilung der Hauptdiagnosen bei der Entlassung ist Tabelle $1 \mathrm{zu}$ entnehmen. Bei den Respondern war die Mehrzahl der Patienten (47,9\%) psychisch krank, besonders im Sinne affektiver Störungen (31,7\%). An der zweiten Stelle befanden sich chronische Erkrankungen des Bewegungsapparats (17,3\%). Weitere Schwerpunkte waren Onkologie $(12,4 \%)$ sowie chronische Schmerzen $(8,0 \%)$.

\section{Gesundheitsbezogene Lebensqualität}

In Tabelle 2 sind Daten über die erfolgten Erhebungen der gesundheitsbezogenen LQ bei allen Respondern zusammengefasst. Es wurden jeweils die Mittelwerte der Stichproben und deren Differenz mit SD sowie die statistische Signifikanz p dargestellt. Die drei erhobenen Werte (Eintritt, Austritt und 4 Wochen poststationär) wurden paarweise statistisch ausgewertet, sodass vorerst eine Aussage bezüglich des Behandlungsergebnisses beim Austritt und im weiteren Vergleich poststationär nach 4 Wochen getroffen werden konnte. Darüber hinaus wurden die LQWerte beim Austritt und nach 4 Wochen miteinander verglichen, um die Veränderung bzw. den kurzfristigen

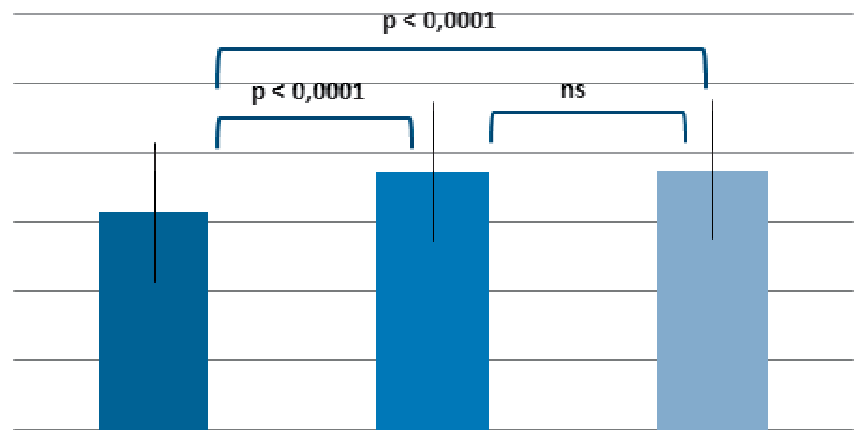

Abb. 1. Erhebungsdaten für die körperliche Gesundheit (KSS).

Hafteffekt des erzielten Erfolgs zu beurteilen. Zusätzlich wurden für sämtliche paarweise verglichenen Parameter die Effektgrösse und der Prozentsatz der Patienten angegeben, die eine Verbesserung der KSS und PSS um mehr als 5 Skalenpunkte erreichten.

Bei Austritt bzw. 4 Wochen danach liessen sich im SF12 ein mittlerer Anstieg für die körperliche Komponente (KSS) um 5,83 bzw. 7,03 und für die psychische Komponente (PSS) um 9,3 bzw. 9,9 Punkte beobachten. Über 60\% aller Patienten erzielten am Ende der Beobachtung Anstiege von mehr als 5 Skalenpunkten. Die Effektgrössen lagen bei 0,68 für die körperliche LQ bzw. 0,87 für die psychische LQ.

In Abbildung 1 werden die Erhebungsdaten für die körperliche Gesundheit (KSS), in Abbildung 2 jene für die psychische Gesundheit (PSS) und in Abbildung 3 die entsprechenden Effektgrössen graphisch dargestellt.

\section{Diskussion}

In der vorliegenden Arbeit wird im Rahmen einer nicht kontrollierten, prospektiven Beobachtungsstudie das subjektive Ergebnis im Sinne von gesundheitsbezogener LQ bei chronisch Kranken während einer stationären Intervention mit integrativer Medizin untersucht. Das ange- 


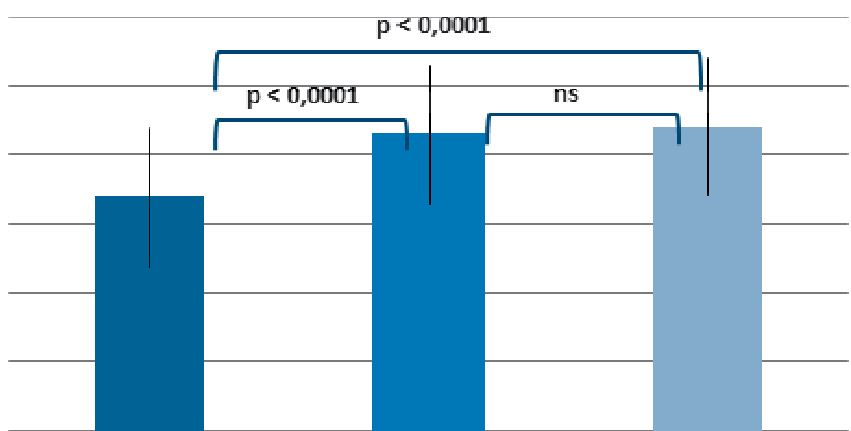

Abb. 2. Erhebungsdaten für die psychische Gesundheit (PSS).

wandte Behandlungskonzept zeichnet sich durch eine individuelle Kombination von Methoden der konventionellen und komplementären Medizin unter gezielter Berücksichtigung gewisser gesundheitspädagogischer Aspekte wie körperliche Aktivität, Ernährung und Entspannungsverfahren aus. Ziel dieser Arbeit war, die subjektiv empfundene Verbesserung der LQ unter diesem multimodalen Therapiekonzept zu quantifizieren. Die Messung von Outcome-Parametern wie Lebensqualität oder Befindlichkeit haben sich für die Erfolgsbeurteilung diverser therapeutischer Massnahmen vor allem im rehabilitativen und psychosomatischen Bereich etabliert [9].

Unsere Ergebnisse zeigen, dass es während einer stationären Behandlung mit integrativem Therapieansatz zu einer wesentlichen Verbesserung sowohl der körperlichen als auch der psychischen Lebensqualität kommt und dass dieser positive Effekt 4 Wochen nach der Entlassung tendenziell weiter zunimmt. Besonders in Bezug auf die psychische Gesundheit ist der Effekt bei den untersuchten Patienten stark ausgeprägt. So steigen die Parameter für die körperliche LQ (KSS) poststationär um 7,03 \pm 9,9 und für die psychische LQ (PSS) um 9,9 $\pm 14,4$ Skalenpunkte. Die entsprechenden Effektgrössen betragen 0,68 bzw. 0,87. Ein Anstieg der SF-12-Parameter um mehr als 5 Skalenpunkte wird bei 61,9 bzw. 67,6\% der untersuchten Patienten erzielt - dieser wesentliche Anstieg ist erfahrungsgemäss mit einem klinisch relevanten Ergebnis verbunden. Darüber hinaus zeigt die Analyse der Ausgangswerte der Patienten einen initial hohen Beschwerdegrad bzw. Leidensdruck - dies unterstreicht die Bedeutung der erzielten Ergebnisse.

Die präsentierten Daten stehen im Einklang mit Resultaten anderer Outcome-Studien in komplementärmedizinischen Krankenhäusern. Diverse klinische Studien konnten belegen, dass stationäre Behandlungen mit einem naturheilkundlichen [10-15] oder integrativen [1619] Ansatz zu einer wesentlichen und nachhaltigen Verbesserung der gesamten LQ führen.

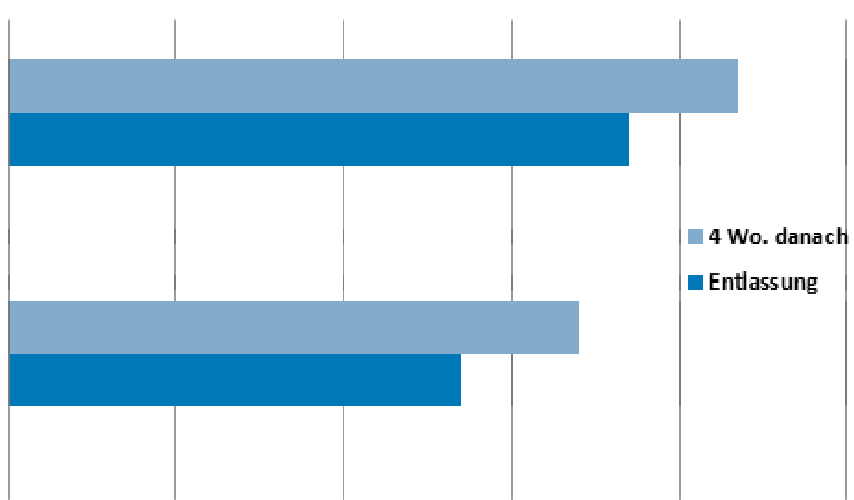

Abb. 3. Effektgrössen aller erhobenen Patienten bei der Entlassung und 4 Wochen poststationär.

Im Vergleich zu diesen Studien war die Anzahl der von uns untersuchten Patienten niedrig $(\mathrm{n}=194)$, was sich durch mehrere Faktoren erklären lässt. Mit 26 stationären Betten kann die private AK nicht mit universitären Einrichtungen wie z.B. der Klinik für Naturheilkunde und Integrative Medizin der Kliniken Essen-Mitte, Deutschland [16, 17], oder Rehabilitationskliniken wie z.B. der Reha-Fachklinik, Bad Elster, Deutschland [10, 15], verglichen werden. Obwohl die AK im Schweizer Gesundheitswesen vollständig integriert ist, werden die notwendigen Kostengutsprachen für eine integrative Behandlung von den Versicherungen sehr restriktiv erteilt. Darüber hinaus müssen gewisse komplementärmedizinische Massnahmen wie Sauerstofftherapien oder Hyperthermien von den Betroffenen direkt bezahlt werden. Diese ökonomischen Aspekte schränken die Aufnahmen ein, obwohl die Kriterien für eine Spitalbedürftigkeit medizinisch erfüllt sind. Die vergleichbar niedrige Anzahl von stationären Patienten $(\mathrm{n}=144)$ in der Erhebung von Heusser at al. [19] lässt auf eine ähnliche Problematik in der Rekrutierung von stationären Patienten in den anthroposophischen Kliniken in der Schweiz schliessen.

Die Rücklaufquote von $83 \%$ für die Fragebögen während der Hospitalisation liegt im Bereich anderer Studien. Ostermann et al. [11] erzielten einen Rücklauf von 82,7\%, Weidenhammer et al. [15] von 80,0\% und Melchart et al. [13] von 84,5\%. 4 Wochen nach der Entlassung schickten nur 54,1\% unserer Patienten den dritten Fragebogen ausgefüllt zurück. Diese nachlassende Beteiligung wird in fast allen vergleichbaren Publikationen beobachtet und ist bei Patienten der AK tendenziell stärker ausgeprägt. Darüber hinaus waren $17 \%$ der in die Beobachtung eingeschlossenen Patienten nicht dazu bereit, sich einer Erhebung zu unterziehen (Non-Responder). Diese Patienten rekrutierten sich vor allem aus dem onkologischen Bereich (45,5\%) und wurden vorwiegend palliativ behandelt - mit entsprechend hoher Mortalität von 27,2\% poststationär. 
Unser Patientenprofil unterschied sich von Studienkollektiven aus vergleichbaren Studien nicht nur durch einen hohen Anteil an onkologischen Leiden (12,4\%), sondern auch durch viele psychiatrische und psychosomatische Krankheiten (47,9\%). Die häufigste Indikation für eine stationäre Behandlung in den bereits zitierten Studien stellten Schmerzen und rheumatische Erkrankungen dar. Diese waren in unserem Patientengut mit 25,3\% eher untervertreten.

Die initiale SF-12-Bewertung unserer Patienten zeigte, dass sich die Hospitalisierten in einem schlechten körperlichen und psychischen Zustand befanden. Der mittlere KSS-Wert von 31,5 bzw. PSS-Wert von 33,9 bei Eintritt befand sich wesentlich unter dem Niveau der entsprechenden Normstichproben für chronisch Kranke. Je nach Krankheitsbild betrugen diese Referenzwerte 40,9-46,2 für KSS und 42,9-51,1 für PSS [6]. Der Vergleich mit anderen, bereits erwähnten Outcome-Studien bestätigt ebenfalls den hohen Beschwerdegrad von untersuchten Patienten. Die Aufnahmewerte für KSS in diesen Studien befanden sich zwischen 33,9 [16] und 40,6 [15], jene für PSS lagen zwischen 36,0 [12] und 41,2 [16]. Somit deuten die um mindestens 2 Skalenpunkte niedrigeren Scores für Patienten der AK auf eine beträchtliche Fallschwere hin. Die Vorgeschichte unserer Patienten war mit 9,2 \pm 7,8 Jahren entsprechend lang. Interessanterweise betrug im gesamten Patientenkollektiv der Migräne-Klinik, Königstein, Deutschland, die mittlere Erkrankungsdauer sogar $23 \pm 12,8$ Jahre [12]. In den übrigen Studien sind die Vorgeschichten der gemischten Patientenkollektive vergleichbar lang. Diese Angaben bestätigen die Beobachtung anderer Autoren, dass komplementärmedizinische Angebote eher von Patienten mit komplexen (Polymorbidität) und lang anhaltenden Leiden (Chronizität) in Anspruch genommen werden $[1,19,20]$.

\section{Ergebnis der integrativen Behandlung}

Während der stationären Behandlung unter integrativer Behandlung kam es im gesamten untersuchten Kollektiv sowohl im Sinne der körperlichen als auch der psychischen Gesundheit zu einer wesentlichen Verbesserung. Der initiale KSS-Wert stieg statistisch signifikant beim Austritt um 5,83 \pm 10,1 und 4 Wochen danach um 7,03 \pm 9,9 Skalenpunkte an. Hinsichtlich der psychischen Gesundheit stieg der PSS-Wert um 9,3 $\pm 12,6$ bzw. um 9,9 \pm 14,4 an. Die poststationären Werte nach 4 Wochen waren tendenziell höher als jene bei der Entlassung, erreichten jedoch nicht statistische Signifikanz. Die beobachteten Effektgrössen zeigten nicht nur eine wesentliche Verbesserung der erhobenen SF-12-Parameter beim Austritt (für KSS 0,54, p $<0,0001$; für PSS $0,74, p<0,0001$ ), sondern auch einen weiteren Zuwachs dieser Indizes 4 Wochen nach der Entlassung (KSS: 0,68, p < 0,0001; PSS: 0,87, p <
0,0001). Alle diese Parameter deuten auf einen mittleren und im Bereich der Psyche sogar starken therapeutischen Effekt der integrativen stationären Behandlung. Diese positiven Effekte liessen sich - gemessen an Wert-Anstiegen von mindestens 5 Skalenpunkten - bei über $60 \%$ der Patienten feststellen (KSS 61,9\%; PSS 67,6\%). Tendenziell waren die Ergebnisse im psychischen Bereich besser als im körperlichen.

Diese Ergebnisse sind grundsätzlich konform mit Beobachtungen anderer Studien mit ähnlichem Design, wobei es auffällt, dass sich die in der AK erreichten Resultate im oberen Bereich der beobachteten Effekte befinden (höhere Effektgrösse). Eine vor kurzem erschienene Metaanalyse von acht Studien bei stationären Patienten mit diversen naturheilkundlichen Ansätzen verschafft einen Überblick über die festgestellten Effektstärken [18]. In der Mehrzahl der eingeschlossenen Studien wurden die Patienten einem «klassischen naturopathischen» Behandlungsansatz unterzogen, bei dem Hydrotherapie, Phytotherapie, Ernährung sowie Lifestyle-Massnahmen im Sinne der Kneipp'schen Medizin zur Anwendung kamen. Zwei Studien untersuchten den Mind-Body-Ansatz. Die erreichten Resultate (gemessen mit SF-36) variierten zwischen einem geringen $(d=0,16)$ und einem moderaten $(d$ = 0,51-0.69) Effekt. Die Effekte fielen bezüglich der psychischen LQ mit einem maximalen Wert von 0,69 höher aus als für die körperliche Gesundheit. Dieses Phänomen haben wir in unserem Patientenkollektiv ebenfalls festgestellt. In der grössten der analysierten Studien waren die Effektgrössen zum Zeitpunkt der Entlassung höher $(0,86)$ als bei dem Follow-up nach 6 Monaten $(0,62)$ [15]. Die allmähliche Verschlechterung des gesundheitlichen $\mathrm{Zu}$ stands der entlassenen Patienten wird regelmässig beobachtet (Auswascheffekt) $[11,13,15-17,19]$ und könnte den relativ hohen Effekt unserer Patienten bei der Entlassung erklären. Das Follow-up unserer Patienten betrug nur 4 Wochen. Dennoch lassen sich zwei Gründe nennen, die dieses gute Ergebnis plausibel machen. Zum Ersten war die Hospitalisationsdauer in der AK $(21,4 \pm 9,8$ Tage $)$ eher länger als in den zitierten Studien (14,7-21,8 Tage). Zum Zweiten scheint unser Therapiekonzept intensiver und komplexer unter besonderem Einsatz von Methoden wie Sauerstofftherapie oder Hyperthermie zu sein. Die Nachhaltigkeit dieses integrativen Behandlungskonzepts müsste noch im weiteren Verlauf nach 6 und 12 Monaten verifiziert werden.

Limitierend für die Aussagen der vorliegenden Arbeit sind mehrere Faktoren. Im Vergleich zu anderen Studien war die Fallzahl der untersuchten Patienten eher niedrig - beispielsweise betraf die Untersuchung von Weidenhammer et al. [15] über 4000 Patienten. Die Anzahl der Patienten, die nicht dazu bereit oder imstande waren, die Fragebögen zu beantworten (Non-Responder), war mit $17 \%$ eher hoch. Diese Patientengruppe war durch ein fort- 
geschrittenes, vorwiegend onkologisches Krankheitsbild mit hoher Mortalität gekennzeichnet. Möglicherweise hätten die Ergebnisse dieser Patienten die gesamte Aussage der Untersuchung im Sinne von geringeren Heilungseffekten verändert. Obgleich sowohl die Entlassungswerte als auch die poststationären Werte nach einem Monat im Vergleich zu anderen Studien höher ausgefallen sind, fehlt es an einem langfristigen Followup. Dies erlaubt keine verlässliche Aussage bezüglich der Nachhaltigkeit der erzielten Resultate.

\section{Schlussfolgerung}

Die Ergebnisse der vorliegenden Beobachtungsstudie zeigen, dass eine stationäre Behandlung mit einem integrativen Therapieansatz zu einem relevanten klinischen
Erfolg mit wesentlicher Verbesserung der körperlichen und psychischen Lebensqualität führt. Dieser Effekt ist 4 Wochen poststationär stärker ausgeprägt als bei der Entlassung, was auf einen mindestens kurzfristigen Hafteffekt der erzielten Ergebnisse deutet. Des Weiteren scheinen die längere Aufenthaltsdauer sowie das intensive und komplexe Behandlungskonzept zu einem tendenziell besseren Endergebnis zu führen. Die getroffenen Aussagen sind vor allem durch die geringe Patientenzahl, den fehlenden Kontrollarm, den Datenverlust durch Non-Responder und die kurze Zeit des Follow-ups limitiert.

\section{Disclosure Statement}

Keine Interessenkonflikte.

\section{Literatur}

1 Astin JA: Why patients use alternative medicine: results of a national study. JAMA 1998 279:1548-1553.

$\checkmark 2$ Kienle GS, Albonico HU, Fischer L, et al: Complementary therapy systems and their integrative evaluation. Explore (NY) 2011;7: 175-187.

3 Heller R, Wiederhöfer D: Zunahme der Relevanz der Ergebnisqualität in der Schweiz; in Oggier W, Walter A, Reichlin S, Egli M (Hrsg): Gesundheitswesen Schweiz im Umbruch. Sursee, Trend Care AG, 2008.

4 Bowling A: Measuring Disease, ed 2. Buckingham, Open University Press, 2001, pp $1-19$.

5 Testa MA, Simonson DC: Assessment of quality-of-life outcomes. N Engl J Med 1996; 334:835-840.

6 Bullinger M: Lebensqualitätsforschung. Bedeutung - Anforderung - Akzeptanz. Stuttgart, Schattauer, 1997, pp 1-7.

7 Morfeld M, Kirchberger I, Bullinger M: SF36. Fragebogen zum Gesundheitszustand, ed 2. Göttingen, Hogrefe, 2011.

-8 Dunlop WP, Cortina JM, Vaslow JB, Burke MJ: Meta-analysis of experiments with matched groups or repeated measures designs. Psychol Methods 1996;1:170-177.

9 Grill TM, Feinstein AR: A critical appraisal of the quality of life measurements. JAMA 1994; 272:619-626.
Beer AM, Ostermann T, Matthiessen PF: Evaluation stationärer Naturheilkunde - das Blankensteiner Modell. Teil I: Patientenklientel und therapeutische Konzepte. Forsch Komplementärmed Klass Naturheilkd 2001; 8:6-13.

11 Ostermann T, Beer AM, Matthiessen PF: Evaluation stationärer Naturheilkunde - das Blankensteiner Modell. Teil II: Effektstärken und Gesundheitsstatus der Patienten im zeitlichen Verlauf. Forsch Komplementärmed Klass Naturheilkd 2002;9:269-276.

12 Melchart D, Weidenhammer W, PollokMüller A, et al: Ergebnisqualität bei der Behandlung chronischer Kopfschmerzpatienten in einer Migräneklinik. Forsch Komplementärmed Klass Naturheilkd 2001;8:219-227.

13 Melchart D, Weidenhammer W, Linke K, et al: 'Quality Profiling' for complementary medicine: the example of a hospital for traditional Chinese medicine. J Altern Complement Med 2003;9:193-206.

14 Melchart D, Wessel A, Wunderlich S: Qualitätsprofil einer Rehabilitationsfachklinik für Naturheilverfahren - Teil 1: Struktur Qualitätssicherung und Interventionen. Forsch Komplementärmed 2007;14:281-288.

15 Weidenhammer W, Wessel A, Melchart D: Qualitätsprofil einer Rehabilitationsfachklinik für Naturheilkunde - Teil 2: Patientenund Ergebnisprofil. Forsch Komplementärmed 2007;14:335-345.
16 Hoffmann B, Moebus S, Michalsen A, et al: Gesundheitsbezogene Kontrollüberzeugung und Lebensqualität bei chronisch Kranken nach stationärer Behandlung mit Integrativer Medizin - eine Beobachtungsstudie. Forsch Komplementärmed Klass Naturheilkd 2004; 11:159-170.

17 Lauche R, Cramer H, Moebus S, et al: Results of a 2-week inpatient stay at the department for internal and integrative medicine: an observational study. Evid Based Complement Alternat Med 2012;2012:875874.

18 Ostermann T, Langhorst J, Beer AM: The effects of integrative in-patient treatment on patients' quality of life: a meta-analysis. Evid Based Complement Alternat Med 2013;2013: 416510.

19 Heusser P, Braun SB, Bertschy M, et al: Palliative in-patient cancer treatment in an anthroposophic hospital: II. Quality of life during and after stationary treatment, and subjective treatment benefits. Forsch Komplementmed 2006;13:156-166.

20 Mermod J, Fischer L, Staub L, Busato A: Patient satisfaction of primary care for musculoskeletal diseases: a comparison between Neural Therapy and conventional medicine. BMC Complement Altern Med 2008;8:33. 